OPEN ACCESS

Edited by:

Mohammad Shamsul Ola,

King Saud University, Saudi Arabia

Reviewed by:

Ilaria Piano,

University of Pisa, Italy

Ilaria Bellezza,

University of Perugia, Italy

*Correspondence: Lalit Pukhrambam Singh

ak1157@wayne.edu;

plsingh@med.wayne.edu

Specialty section:

This article was submitted to

Neurodegeneration,

a section of the journal

Frontiers in Neuroscience

Received: 31 July 2019

Accepted: 24 September 2019

Published: 10 October 2019

Citation:

Yumnamcha T, Devi TS and

Singh LP (2019) Auranofin Mediates

Mitochondrial Dysregulation

and Inflammatory Cell Death

in Human Retinal Pigment Epithelial

Cells: Implications of Retinal

Neurodegenerative Diseases.

Front. Neurosci. 13:1065.

doi: 10.3389/fnins.2019.01065

\section{Auranofin Mediates Mitochondrial Dysregulation and Inflammatory Cell Death in Human Retinal Pigment Epithelial Cells: Implications of Retinal Neurodegenerative Diseases}

\section{Thangal Yumnamcha, Takhellembam Swornalata Devi and Lalit Pukhrambam Singh* \\ Department of Ophthalmology, Visual and Anatomical Sciences (OVAS), Wayne State University School of Medicine, Detroit, MI, United States}

Purpose: Photoreceptor degeneration occurs in various retinal diseases including age-related macular degeneration (AMD), Retinitis pigmentosa (RP), and diabetic retinopathy (DR). However, molecular mechanisms are not fully understood yet. The retinal pigment epithelium (RPE) forms the outer blood retinal barrier (oBRB) and supplies glucose, oxygen and nutrients from the fenestrated choriocapillaris to photoreceptors for visual function. Therefore, RPE dysfunction leads to photoreceptor injury/death and progression of blinding eye diseases. This study aims to understand the role of the thioredoxin (Trx) and its reductase (TrxR) redox signaling in human RPE dysfunction and cell death mechanism(s) in an in vitro system.

Methods: A human RPE cell line (APRE-19) was cultured in DMEM/F12 medium and treated with auranofin (AF - $4 \mu \mathrm{M}$, an inhibitor of TrxR) for 4 and $24 \mathrm{~h}$. Mitochondrial and lysosomal function, cellular oxidative stress and NLRP3 inflammasome activity were measured using cell assays, Western blotting, and confocal microscopy. Antioxidants and anti-inflammatory compounds were tested for blocking AF effects on RPE damage. Cell death mechanisms ( $\mathrm{LDH}$ release to culture media) were determined using necroptosis, ferroptosis and pyroptosis inhibitors. $P<0.05$ was considered significant in statistical analysis.

Results: Auranofin causes mitochondrial dysfunction $(\Delta \psi \mathrm{m} \downarrow$ and ATP $\downarrow)$, oxidative stress $\left(\mathrm{H}_{2} \mathrm{O}_{2} \uparrow\right)$ and mitophagic flux to lysosomes. Furthermore, the lysosomal enzyme (cathepsin L) activity is reduced while that of pro-inflammatory caspase-1 (NLRP3 inflammasome) is enhanced in ARPE-19. These effects of AF on ARPE-19 are inhibited by antioxidant N-acetylcysteine ( $5 \mathrm{mM}, \mathrm{NAC}$ ) and significantly by a combination of SS31 (mitochondrial antioxidant) and anti-inflammatory drugs (amlexanox and tranilast). AF also causes cell death as measured by cytosolic LDH release/leakage, which is not inhibited by either ferrostatin-1 or necrostatin-1 (ferroptosis and necroptosis inhibitors, respectively). Conversely, AF-induced LDH release is significantly reduced by MCC950 and Ac-YVAD-cmk (NLRP3 and Caspase-1 inhibitors, respectively), suggesting a pro-inflammatory cell death by pyroptosis. 
Conclusion: The Trx/TrxR redox system is critical for RPE function and viability. We previously showed that thioredoxin-interacting protein (TXNIP) is strongly induced in DR inhibiting the Trx/TrxR system and RPE dysfunction. Therefore, our results suggest that the TXNIP-Trx-TrxR redox pathway may participate in RPE dysfunction in DR and other retinal neurodegenerative diseases.

Keywords: neurodegeneration, mitophagy, auranofin, Trx-TrxR, pyroptosis, inflammation, RPE

\section{INTRODUCTION}

Retina is a window to the brain (Chiquita et al., 2019). Being a part of the central nervous system, the retina consumes large amounts of glucose and oxygen for its bioenergetics (ATP production), light perception, and visual processing (Country, 2017). The retina also has tight blood-retinal barriers (BRB) and protects the neuroretina from the circulating immune cells and plasma components (Cunha-Vaz et al., 2011). The inner BRB consists of tight junctions of endothelial cells in the blood vessel while the outer BRB consists of a single layer of retinal pigmented epithelium (RPE) and its tight junction proteins. RPE separates the fenestrated choriocapillaris from the neuroretina and functions to transport glucose, oxygen, and nutrients to the retinal outer segments consisting of rod and cone photoreceptors (Campbell and Humphries, 2012; Ivanova et al., 2019). Breakdown of the outer BRB and RPE dysfunction is associated with age-related macular degeneration (AMD) (Handa et al., 2019; Jun et al., 2019) while gene mutation in RPE and photoreceptor cause photoreceptor degeneration and blindness including retinitis pigmentosa (RP) (Dias et al., 2018; Shu and Dunaief, 2018). In these various retinal diseases, RPE dysfunction, photoreceptor injury/death and retinal neurodegeneration leads to blindness. In addition to nutrient exchange, RPE also involves in recycling of the visual pigment (retinoid) to photoreceptors, daily phagocytosis of the photoreceptor outer segment, and synthesis of melanosome (melanin) to absorb excess light in the retina (Biesemeier et al., 2015; Spencer et al., 2019).

On the other hand, breakdown of the inner BRB and formation of new fragile/leaky blood vessels are associated in proliferative diabetic retinopathy (PDR) leading to blindness as well as visual distortion in diabetic macular edema (DME) (Graham et al., 2018; Bapputty et al., 2019). Recent studies have further demonstrated that photoreceptor oxidative stress and dysfunction may occur early in diabetics prior to vessel histopathology (Fu et al., 2018; Liu et al., 2019). However, the study of the role of RPE in photoreceptor dysfunction in DR is still limited (Xia and Rizzolo, 2017; Tarchick et al., 2019). Diabetic retinopathy (DR) is the most devastating disease of diabetes mellitus affecting millions of people among the working adult life in the US and around the globe (Cheloni et al., 2019). As the number of people living with diabetes particularly of obesity and type 2 diabetes increases, the incident of DR will escalate several folds in coming decades (Caspard et al., 2018). DR is generally defined by microvascular complications of capillary endothelium and pericytes leading to microaneurysm, iBRB leakage, and fragile new blood vessel formation (neovascularization).
Most patients with Type 1 or Type 2 diabetes will develop some forms of DR, beginning with non-proliferative DR (NPDR) then progress to a severe form of proliferative DR (PDR) causing blindness (Xia and Rizzolo, 2017; Fu et al., 2018). Yet, the molecular basis of the pathogenesis is not fully understood and, therefore, no known cure or effective treatment options are available still today.

The retina consumes large amounts of glucose and oxygen to generate energy (ATP) for its visual function via the mitochondrial electron transport chain (ETC) in inner membranes (Country, 2017). During this process, electrons leak out from the ETC, which are captured by molecular oxygen generating reactive oxygen radicals/species (ROS), which damage mitochondrial membrane lipid, proteins, and mtDNA. Damaged mitochondrial are inefficient in ATP but produces more ROS. Mitochondrial ROS and oxidized mtDNA, when released into the cytosol, are recognized as damagedassociated molecular patterns (DAMPs) by cytosolic pattern recognition receptors (PRRs) including toll-like receptors TLR4, TLR9, and the NLRP3 inflammasome. These inflammatory receptors produce and activate inflammatory pro-IL-1 $\beta$ and pro-caspase-1. An assembled NLRP3 inflammasome forms the platform for pro-caspase-1 processing and activation, which in turn is responsible for processing pro-inflammatory IL- $1 \beta$ and active IL- $1 \beta$. Caspase- 1 also induces pro-inflammatory cell death by pyroptosis, which involves release of the $\mathrm{N}$-terminal part of gasdermin D (Dubois et al., 2019). The cleaved $\mathrm{N}$-terminus portion of gasdermin $\mathrm{D}$ is then inserted into the plasma membrane forming pores resulting in plasma membrane permeabilization. Therefore, removal of the damaged mitochondrial by mitophagy, an autophagic process of degrading damaged mitochondria by lysosomes, is critical for maintaining mitochondria health, bioenergetics, and cell survival (Devi et al., 2019). In addition, the RPE phagocytes the damaged photoreceptor outer segment daily and recycle visual pigment retinol for photoreceptor function. Therefore, the mitochondriallysosomal axis in RPE and maintaining its function may play a critical role in photoreceptor function in DR. Hence, enhancing the antioxidant capacity while reducing inflammation at initial stages of the disease may constitute potential therapies (Lin and Beal, 2006; Di Carlo et al., 2012; Stephenson et al., 2018; Bapputty et al., 2019).

One of the genes strongly induced by diabetes and aging in retinal cells and neurons is the thioredoxin-interacting/inhibiting protein (TXNIP) (Perrone et al., 2010; Devi et al., 2012, 2013, 2019; Singh and Perrone, 2013). TXNIP's actions include binding to and inhibition of the anti-oxidant and thiol reducing capacity of thioredoxin ( $\operatorname{Trx})$, thereby, causing cellular oxidative stress, 
inflammation, and premature cell death (Stephenson et al., 2018). Trx1 and its reductase TrxR1 are present in the cytosol and nucleus while Trx2/TrxR2 redox system is in the mitochondrion. Therefore, Trx/TrxR redox system plays an important role in scavenging reactive oxygen species (ROS) and maintaining reduced state of proteins in their functionally active sites and cell survival (Devi et al., 2017; Stephenson et al., 2018; Booty et al., 2019). Thus, TXNIP inhibition of the Trx/TrxR system causes oxidative stress, mitochondrial dysfunction, and retinal cell death in diabetes (Perrone et al., 2010; Singh and Perrone, 2013). Nonetheless, TXNIP is a multifunctional protein and is also considered as a scaffold protein of the $\alpha$-arrestin type (Stephenson et al., 2018). Therefore, TXNIP is also involved in interactions with other proteins including glucose transporter 1 and 4, REDD1 (regulated in development and DNA damage responses 1), VEGF-R (vascular endothelial growth factor receptor), and other nuclear proteins that are involved in cell cycle regulation (Devi et al., 2012; Stephenson et al., 2018). Therefore, to further understand a direct effect of the Trx/TrxR redox system in RPE and retinal neurodegenerative diseases, we used in this study, auranofin, a gold compound, which specifically inhibits redox proteins TrxR1 and TrxR2 (Yoshihara et al., 2014). We demonstrate that auranofin causes mitochondrial dysfunction, mitophagic flux and inflammatory pyroptotic cell death in RPE cells in culture. These findings may be relevant to various retinal neurodegenerative diseases where $\mathrm{RPE}$ dysfunction plays a causative role in disease progression.

\section{MATERIALS AND METHODS}

\section{Tissue Culture Media}

DMEM medium was purchased from (Mediatech Inc., Cat \#10014-CM, Manassas, VA, United States) while Ham's F12 was from Hyclone (Cat \# SH30025.01, Logan, UT, United States). Antibiotics and trypsin were also purchased from Hyclone, whereas fetal bovine serum was obstained (Corning, Cat \# MT35010CV). Details of other chemicals used in this study are provided in Supplementary Table S1.

\section{Cell Culture}

A human retinal pigment epithelial cell line (ARPE-19), purchased from ATCC (Cat\# 2302), was maintained in DMEM/F12 medium (1:1 ratio) containing LG (low glucose, $5.5 \mathrm{mM}$ ), 5\% fetal bovine serum (FBS), 2\% penicillin, and $1 \%$ antimycotic in a humidified incubator with $5 \% \mathrm{CO}_{2}$ at $37^{\circ} \mathrm{C}$ as described previously (Devi et al., 2013). After reaching $\sim 80 \%$ confluence, the medium was changed to $1 \%$ serum overnight. Initially, a concentration and time dependent effect of auranofin (AF) on ARPE-19 function was tested; and subsequently we selected $4 \mu \mathrm{M}$ and $4-24 \mathrm{~h}$ as optimal concentration and time period for the current study. Then, ARPE-19 cells were maintained with or without AF $(4 \mu \mathrm{M})$ for 4 and $24 \mathrm{~h}$, respectively. Because we observed cell death at $24 \mathrm{~h}$ but not at $4 \mathrm{~h}$ of AF treatment, we decided to investigate early molecular defects that might lead to demise and determine potential rescue mechanisms. Before treatment with $4 \mu \mathrm{M}$ AF, we pre-incubated
ARPE-19 cells with different concentration of drugs targeting mitochondria and NLRP3 inflammasome for $2 \mathrm{~h}$ and they were present throughout the period of incubation.

\section{Measurement of Mitochondrial Membrane Potential}

The mitochondrial membrane potential in ARPE-19 cells was measured using MitoProbeTM JC-1 Assay Kit (Cat\# M34152, Life Technologies, Eugene, OR, United States) (Devi et al., 2012, 2013). JC1 dye penetrates the cell and exhibits potentialdependent accumulation in mitochondria. After treatment, ARPE 19 cells were washed with $1 \times$ phosphate-buffered saline (PBS). Then, the cells were incubated with $200 \mu \mathrm{l}$ of $2 \mu \mathrm{M}$ JC1 dye for $10 \mathrm{~min}$ in a 48-well cell culture plate. After incubation, the cells were washed thrice with $1 \times$ PBS. The relative fluorescence was measured at Ex529/Em590 nm using (SpectraMax Gemini EM Microplate Reader, Molecular Devices) as per manufacturer's instructions.

\section{Measurement of Intracellular ATP}

Cellular ATP concentration in ARPE-19 cells was measured using a ATP determination kit from Life Technologies (Cat \# A22066, Life Technologies, Eugene, OR, United States) (Devi et al., 2012, 2013). After treatment, ARPE-19 cells were washed twice with $1 \times$ PBS. Then, $250 \mu \mathrm{l}$ of $1 \times$ TE Buffer (Tris EDTA, $1 \times$ solution, $\mathrm{pH}$ 8.0, Cat \#BP24731, Thermo Fisher Scientific) was added to each well of a 48 -well cell culture plate containing the ARPE-19 cells. The cells were scrapped out and transferred to a $1.5 \mathrm{ml}$ Eppendorf tube. The samples were boiled for $5 \mathrm{~min}$ in a water bath. After keeping on ice for $3 \mathrm{~min}$, the samples were centrifuged at $12000 \mathrm{rpm}$ for $10 \mathrm{~min}$ at $4^{\circ} \mathrm{C}$. Ninety $\mu \mathrm{l}$ of the ATP reaction solution and $10 \mu \mathrm{l}$ of the sample were mixed and kept protected from light. Relative fluorescence units (RFUs) were measured using luminometer plate reader (SpectraMax L Microplate Reader, Molecular Devices).

\section{Measurement of Intracellular Reactive Oxygen Species (ROS)}

Cellular ROS production in ARPE-19 cells was measured using the CM-H2DCFDA probe (Cat \# 6827, Life Technologies, Eugene, OR, United States) as per company's instructions. After different treatments, ARPE-19 cells were washed twice with $1 \times$ PBS and incubated with $200 \mu \mathrm{l}$ of $10 \mu \mathrm{M}$ CM-H2DCFDA dye for $30 \mathrm{~min}$ at $37^{\circ} \mathrm{C}$ on 24 -well cell culture plate. Following incubation, ARPE-19 cells were washed once in $1 \times$ PBS. The fluorescence intensity of CM-H2DXFDA was measured at Ex495/Em517 nm in $1 \times$ PBS buffer using a Fluorescence Plate Reader (SpectraMax Gemini EM Microplate Reader, Molecular Devices).

\section{Assessment of Cathepsin-L Activity}

Cathepsin-L activity in ARPE-19 cells was measured using a Magic Red Cathepsin-L detection Kit (Cat \#941, Immunochemistry Technologies, Bloomington, $\mathrm{MN}$, United States) as described recently (Devi et al., 2013). Briefly, ARPE-19 cells were incubated with $20 \mu \mathrm{l}$ Magic Red 
Cathepsin-L reagent and $480 \mu \mathrm{l}$ of cell culture media for $1 \mathrm{~h}$. The cells were then washed twice with $1 \times$ PBS. The fluorescence intensity of dye was measured at Ex592/Em628 nm using a Fluorescence Plate Reader (SpectraMax Gemini EM Microplate Reader, Molecular Devices).

\section{Assessment of Caspase-1 Activity}

Caspase-1 activity in ARPE-19 cells was measured using a FAM-FLICA Caspase-1 detection kit (Cat \#97, Immunochemistry Technologies, Bloomington, $\mathrm{MN}$, United States) in a 24-well cell culture plate as described before (Devi et al., 2013). After treatment with various reagents for a stipulated time period, ARPE-19 cells were washed with wash buffer. Then, the cells were incubated with $10 \mu \mathrm{l}$ of FLICA solution and $300 \mu \mathrm{l}$ of freshly replaced $1 \%$ serum media for $1 \mathrm{~h}$. Following incubation, the cells were washed twice with $1 \times$ PBS. The fluorescence intensity was measured at Ex492/Em520 nm using a Fluorescence Plate Reader (SpectraMax Gemini EM Microplate Reader, Molecular Devices).

\section{Measurement of Lactate Dehydrogenase Activity (LDH)}

Lactate dehydrogenase activity in ARPE-19 cells was measured using Pierce TM LDH cytotoxicity Assay Kit (Cat\# 88954, Thermo Fisher scientific). After treatment, $50 \mu \mathrm{l}$ of the Sample's cell culture media were mixed with $50 \mu \mathrm{l}$ of reaction mixture in a 96-well plate and incubated at room temperature for $30 \mathrm{~min}$. The absorbance was measured at $490 \mathrm{~nm}$ and $680 \mathrm{~nm}$ using SpectraMax plus 384 Microplate Reader (Molecular Devices), as per company's instructions. The amount of $\mathrm{LDH}$ release in the media was calculated by difference in absorbance of 490-680 nm, according to manufacturer's instructions.

\section{Western Blotting and Mitophagic Flux}

These procedures, both Western blotting and mitophagy analysis, were performed similar to those described (Devi et al., 2017, 2019). Cytosol, mitochondria and nuclear fractions were fractionated using a kit as described before (Devi et al., 2012, 2013). Briefly, for Western blots, 20-30 $\mu \mathrm{g}$ protein extracts were loaded on SDS-PAGE, and proteins were separated, transblotted and incubated with appropriate primary antibodies. After overnight incubation with primary antibodies at $40_{\mathrm{c}}$ with shaking, HRP conjugate secondary antibodies were added for $2 \mathrm{~h}$. ECL detected the reactive bands in a FluorChem E Western blot imaging instrument (ProteinSimple, San Jose, California). ImageJ (NIH) was used to quantitate the blots. The source of primary and secondary antibodies and their dilution used are shown in Supplementary Tables S2A,B, respectively.

For mitophagy, Ad-CMV-mt-Keima was expressed in the mitochondrial matrix by transduction for 3 days; then treated with AF with or without inhibitors. Mt-Keima emits a green light (neutral or alkaline $\mathrm{pH}>7.0$ ) in mitochondria while, in lysosomes after mitophagic flux, it emits red light at acidic $\mathrm{pH}$ $(<5.0)$. Therefore, the same mt-Keima can detect mitophagic flux by a change in color from green to red in living cells. A Zeiss LMS 780 Confocal Microscope captured multiple images from triplicate samples. Similarly, a CMV-LAMP1-mCherry carrying adenovirus was transduced in ARPE-19 cells to examine lysosomal morphology and distribution after the AF treatment.

\section{Statistical Analysis}

Results are represented by means \pm SEM of the indicated number of experiments. One-way ANOVA and Bonferroni post hoc test determined differences among means in multiple sets of experiments. On the other hand, a comparison between two sets of experiments was analyzed by unpaired two-tailed $t$-test. A $p$-value of $<0.05$ was considered to be statistically significant.

\section{RESULTS}

\section{Auranofin Causes Mitochondria- Lysosome Dysfunction and Inflammatory Responses in ARPE-19 Cells}

Treatment of APRE-19 with AF $(4 \mu \mathrm{M})$ for $4 \mathrm{~h}$ reduces significantly the mitochondrial ATP level as well as mediates mitochondrial membrane depolarization as measured by a reduction in JC1 (Figures 1A,B, respectively). In addition, there is an increase in the cellular ROS level as determined by $\mathrm{H}_{2}$ DCFDA probe (Figure 1C). These results suggest that AF induces mitochondrial dysfunction and cellular oxidative stress in ARPE-19. To further investigate if lysosomal function is also altered due to mitochondrial defects as the mitochondrialysosome function are closely related, we measured the activity of lysosomal acid hydrolase, cathepsin L. Indeed, we observed that cathepsin L activity is significantly reduced by AF (Figure 1D) and also increases the activity of pro-inflammatory caspase-1 (Figure 1E) suggesting inflammasome activation by the AF treatment. Furthermore, we show that lysosome destabilization by lysosomotropic agent LLME (LeuLeuOMe) also causes a reduction in ATP level along with activation of caspase 1 and inhibition of the cathepsin L activity, which are similar to that observed with AF further supporting that the mitochondria and lysosomal injury in AF action on ARPE-19 cells (Figure 2). Nonetheless, AF does not alter levels of TrxR1 and TrxR2 significantly and similarly no significant change is observed with $\operatorname{Trx} 1$ and $\operatorname{Trx} 2$, although $\operatorname{Trx} 2$ is marginally reduced (Figure 3 ).

\section{Auranofin Does Not Evoke mtUPR but Mediates Mitophagic Flux in ARPE-19 Cells}

The mitochondrion responses to oxidative stress (i) by increasing the expression of nuclear-encoded mitochondria-targeted chaperones and proteases to counter its oxidative protein stress and misfolding known as the mitochondrial unfolded protein response (mtUPR) (Harper, 2019). (ii) Another mitochondrial stress response is segregation of the damaged part of the mitochondrion by fission involving Drp1 (dynamin related protein 1), then engulfment within a double-membrane autophagosome, which is further targeted to lysosomes for degradation, a process known as 
mitophagy - autophagy of damaged mitochondria (Pareek and Pallanck, 2018). Nonetheless, we did not observe significant changes in the expression of mitochondrial proteases (LonP and YMEIL1) and chaperones (Tid1/mtHSP40 and PDIA, protein disulfide isomerase A) by AF. Conversely, during the same period of AF treatment, autophagic/mitophagic markers, such as microtubule light-chain LC3BII and adaptors optineurin and p62/Sequestosome1, are reduced within minutes to hours (Supplementary Figure S1), suggesting a mitophagy induction.

Subsequently, we examined AF-induced mitophagic flux in ARPE-19 cells using a mito-probe known as mt-Keima
(Devi et al., 2013), which emits green light in mitochondria at neutral or alkaline $\mathrm{pH}(>7.0)$ whereas it emits red light after mitophagic flux to lysosomes at acidic $\mathrm{pH}(<5.0)$. Using confocal live cell imaging of ARPE-19 after mt-Keima transduction and treatment with AF, we observed mt-Keima in control cells as green filaments of mitochondria, and a lesser amount of the red mt-Keima (Figure 4A, first panel). Conversely, AF treatment increases the level of red mt-Keima in ARPE-19, indicating a mitophagy flux to acidic lysosomes (Figure 4A, second panel). Next, we tested effectiveness of several inhibitors in combination targeting different steps in the mitochondria-lysosome pathway (Supplementary Figures S2, S3).
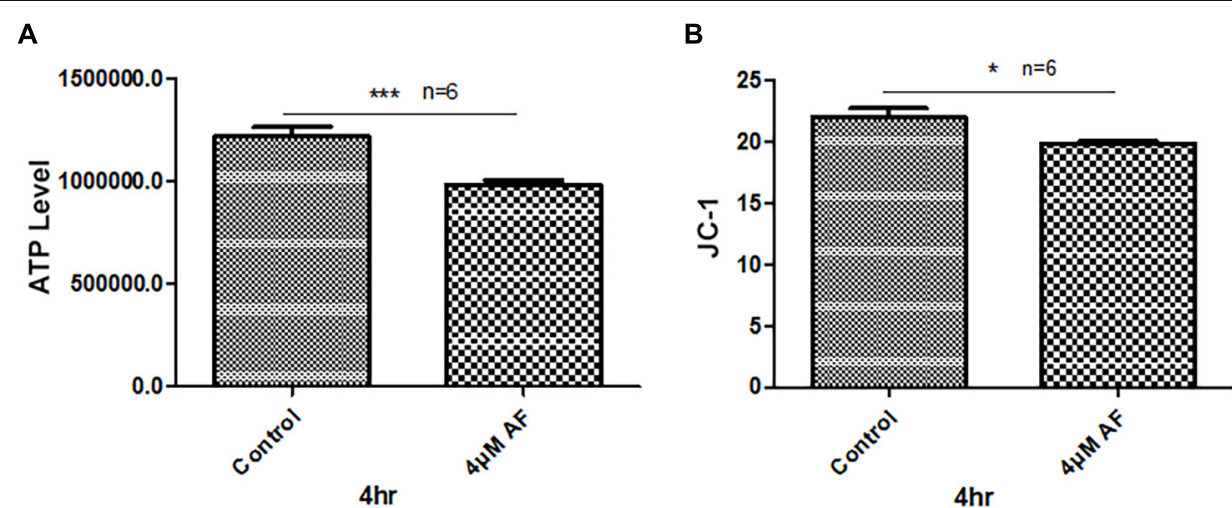

C

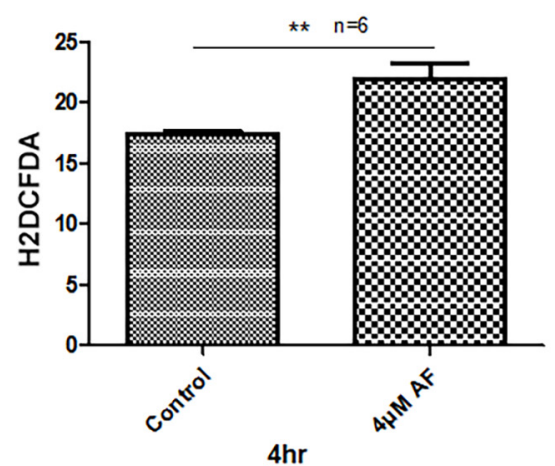

D
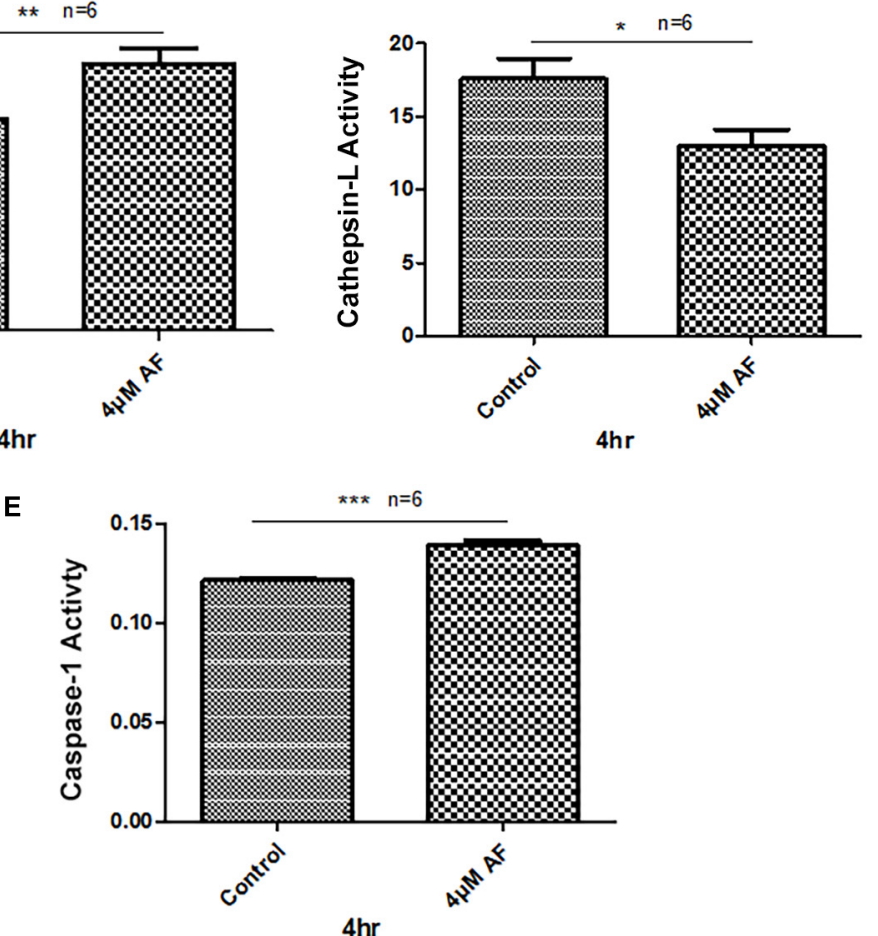

FIGURE 1 | Auranofin causes mitochondrial dysfunction and lysosomal enzyme inactivation in ARPE-19 cells. (A) AF treatment (4 $\mu$ M, 4 h) of ARPE-19 cells reduces cellular ATP level and (B) causes mitochondrial membrane depolarization as measured by a reduction in JC1. (C) In addition, AF increases cellular ROS levels and (D) reduces lysosomal cathepsin $L$ activity. These changes in mitochondrial and lysosomal function are associated with an increase in the activity of proinflammatory enzyme, caspase-1 (E). Significant changes are indicated by $p$ values of ${ }^{*}<0.05 ; * *<0.001$; and ${ }^{* * *}<0.0001 ; n=6$. 

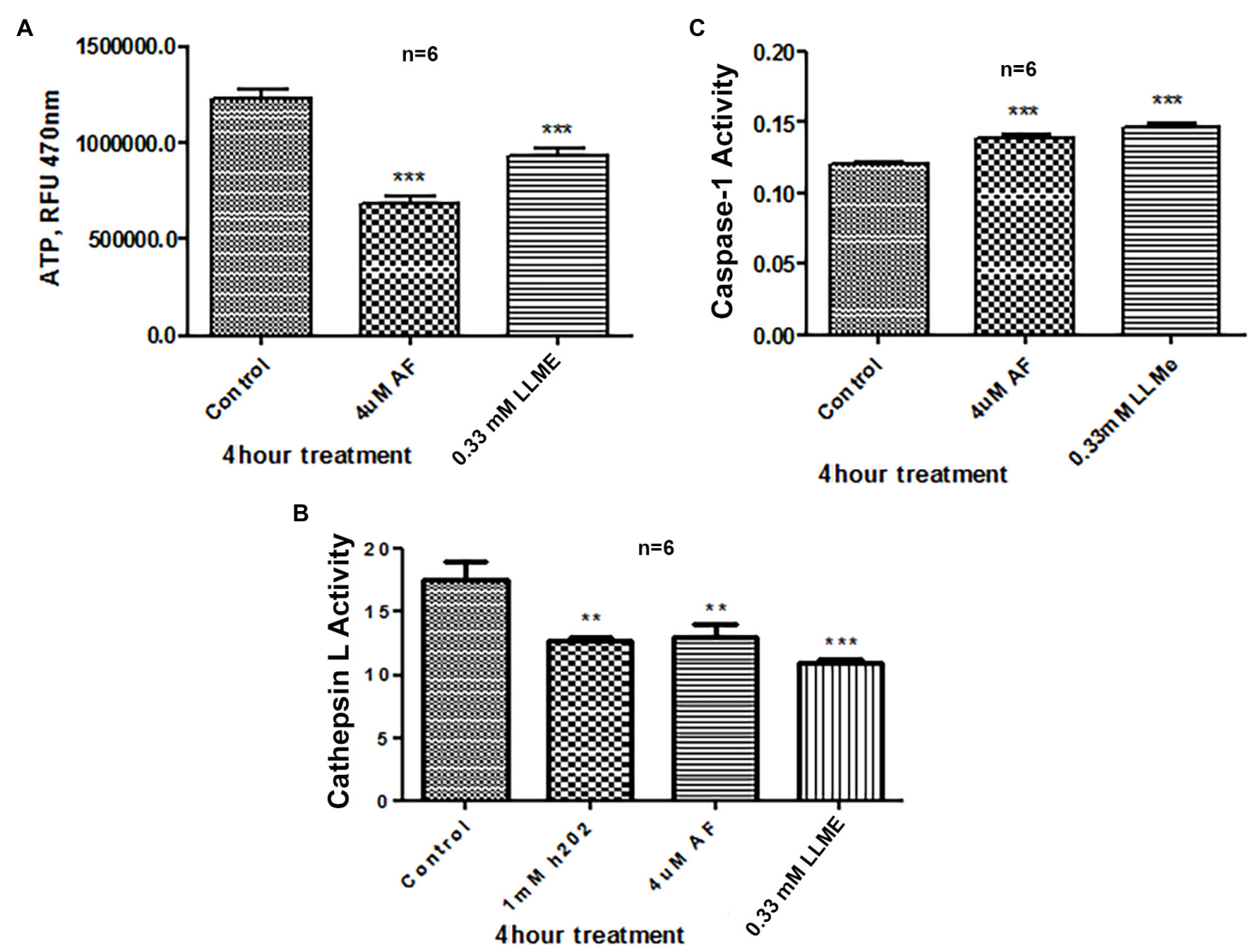

ARPE-19

FIGURE 2 | Lysosomal damage reduces ATP levels and activates Caspase-1 activity in ARPE-19 cells. (A,B) Treatment with auranofin (AF, $4 \mu$ M, 4 h) or lysosomal membrane iononophore (LLMe, $0.33 \mathrm{mM}, 4 \mathrm{~h}$ ) significantly reduces ATP levels and cathepsin $\mathrm{L}$ activity. In addition, $\mathrm{H}_{2} \mathrm{O}_{2}$ also reduces cathepsin $L$ activity significantly suggesting a role for oxidative stress. (C) Conversely, both AF and LLMe increase pro-inflammatory caspase1 activity in ARPE-19 cells. Significant changes in figures are indicated by $p$ values of symbols ${ }^{* *}<0.001$ and ${ }^{* * *}<0.0001 ; n=6$ for each experiment.

These include SS31 - mitochondrial antioxidant (Fivenson et al., 2017), Mdiv-1 - Drp1 fission inhibitor (Campbell et al., 2019), amlexanox - TBK1 and Optineurin/p62 inhibition (Devi et al., 2013; Manczak et al., 2019) and tranilast - NLRP3 inhibitor (Oral et al., 2017). As shown in Figure 4A (last 2 panels), we observe that the presence of Mdiv-1 reduces the level of red mt-Keima of AF but produces vesiculated mitochondria, indicating a prevention of the mitophagic flux and an accumulation of damaged mitochondria. However, a combination of SS31 + Amlexanox + Tranilast prevents AF-induced mitophagic flux as the level of green mt-Keima is maintained and the mitochondria are seen as filamentous networks. In support, the level of autophagic/mitophagic markers, LC3BII and Optineurin proteins, are increased in the present of Mdiv-1 suggesting mitophagy inhibition and reduced lysosomal degradation, which is not observed with SS31 + Amlexanox + Tranilast (Supplementary Figure S4). Furthermore, this later three drug combination normalizes mitochondrial ATP level, which is also seen with anti-oxidant NAC (N-acetylcysteine) (Figure 5), again suggesting a role for oxidative stress in mitochondrial dysregulation in ARPE-19.
Therefore, this drug combination may have potential therapeutic benefits in neurodegenerative diseases.

\section{AF Causes Nuclear Deformation and Transcription Factor EB Translocation in ARPE-19 Cells}

An interesting observation with AF treatment in ARPE-19 is that the perinuclear DNA shrinks and deform when compared with the control suggesting nuclear stress (shown in Figure 4B). However, AF effects on nuclear deformation are restored by combination treatment with SS31 + Amlexanox + Tranilast, but not in the presence of Mdiv-1 (Figures 4B,C). Furthermore, AF treatment causes lysosomal destabilization shown by peripheral LAMP1-mCherry expression and membrane exocytosis in ARPE-19 cells (Supplementary Figure S5). Subsequently, one of the lysosomal membrane-associated transcription factors, TFEB, migrates from the lysosome to the nucleus as a mitophagy-lysosomal stress response (Figures 6A-C). In fact, the combination treatment with SS31 + Amlexanox + Tranilast also restores AF-induced TFEB 


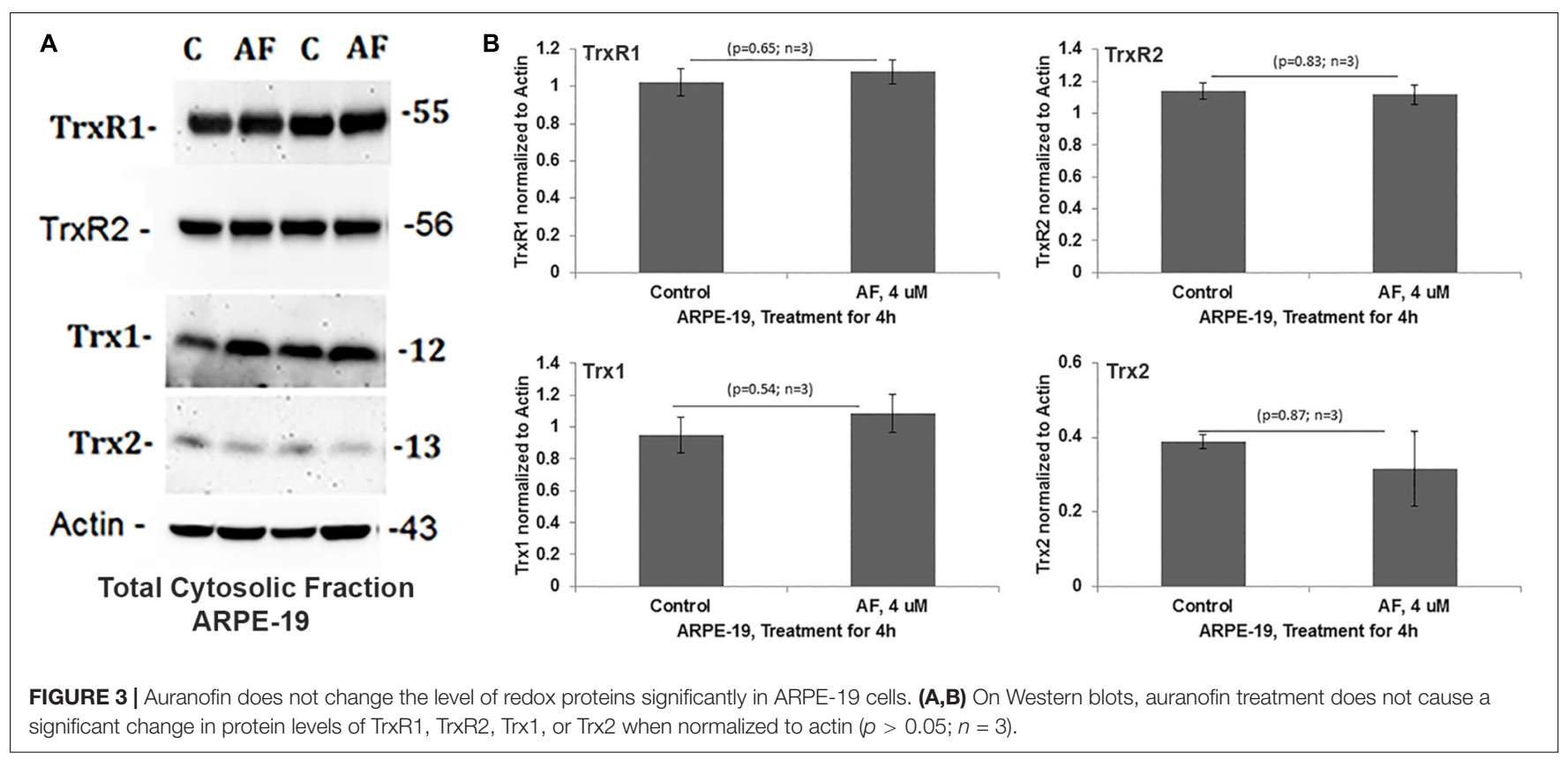

nuclear translocation (Supplementary Figure S6), suggesting lysosomal stabilization. TFEB is involved in the synthesis of lysosomal and autophagy genes within a gene network known as the coordinated lysosomal expression and autophagy regulation (CLEAR) (Huang et al., 2018; Pan et al., 2019). On the other hand, p53 also known to response to DNA damage and cellular stress is not altered by AF treatment. However, AF treatment significantly reduces the level of nuclear actin (Figures 6A,B). Nuclear actin filaments together with lamins form the peripheral nuclear skeletal network; therefore, this observation may be related to nuclear DNA deformation or shrinkage as described above (Figure 4B) and may affect transcriptional network of TFEB.

\section{AF Induces Pro-inflammatory Pyroptotic Cell Death in ARPE-19 Cells}

To examine further that AF causes ARPE-19 cells death, we used the release of cytosolic $\mathrm{LDH}$ to the culture media upon plasma membrane leakage. We did not observe ARPE-19 cell death at $4 \mathrm{~h}$ although mitochondrial dysregulation occurs as demonstrated above. However, as shown in Figures 6A, 7A AF increases $\mathrm{LDH}$ release at $24 \mathrm{~h}$ indicating ARPE-19 demise, which is significantly reduced by $2 \mathrm{~h}$ pre-incubation of a combination of SS31 + Amlexanox + Tranilast before auranofin. However, the AF-mediated LDH release is not prevented by ferrostatin 1, an inhibition of ferroptosis (Settembre and Medina, 2015), while NAC prevents cell death indicating oxidative stress mechanisms (Figure 7B). Ferroptosis is a newly identified cell death mechanism due to iron-accumulation and lipid peroxidation and inactivation of glutathione peroxidase 4 (GPX4), which detoxifies membrane lipid peroxidation (Yoshida et al., 2019). Similarly, necrostatin 1, an inhibitor of necroptosis (Forcina and Dixon, 2019), has no effect on AF-mediated ARPE-19 death (Figure 7C). On the other hand, inhibition of NLRP3 inflammasome and caspase-1 together (MCC950 and Ac-YVAD-cmk, respectively) reduces AF-mediated LDH leakage significantly in ARPE-19 (Figure 7D), indicating an inflammatory cell death mediated by caspase-1 known as pyroptosis (Hanus et al., 2016). Because mitochondria are critical for iron metabolism and its TCA cycle enzymes and ETC complexes contain iron-sulfur complexes (Pronin et al., 2019), when fluxed to lysosomes via mitophagy, there may be iron accumulation and ferroptosis. Therefore, to ensure that AF-induced ARPE-19 demise is indeed due to pyroptosis, but not by ferroptosis under the experimental conditions, we again treated ARPE-19 cells with RSL3, an inducer of ferroptosis (Lill et al., 2012). Indeed RSL3 induces LDH release in ARPE-19, which is prevented by ferrostatin 1 (Figure 7E), indicating that ferroptosis occurs in RPE cells. Furthermore, AF increases the level of gasdermin D (a pyroptosis marker) in ARPE-19 at 4 h significantly ( $p<0.05$ vs. control, 0 time), then returns to the basal level at $24 \mathrm{~h}$ (Supplementary Figure S7). Cytosolic gasdermin D is cleaved by activated caspase- 1 and then inserts into the plasma membrane to form pores and leakage (Dubois et al., 2019). Thus, the results here suggest that AF causes cellular oxidative stress, mitochondria-lysosome dysregulation, and inflammatory pyroptotic cell death in ARPE-19.

\section{DISCUSSION}

The current study identifies that the Trx redox system is critical for RPE function and an inhibition of the Trx/TrxR thiol redox by AF leads to cellular oxidative stress, mitochondrial damage, lysosomal dysfunction, and pro-inflammatory cell death as summarized in Figure 8. Furthermore, we recently published that hyperglycemia-induced TXNIP upregulation in RPE cells where Trx 1 and Trx 2 are inhibited causes mitochondrial damage, mitophagic flux to lysosomes and lysosomal enlargement in human RPE cells under diabetic conditions (Devi et al., 2019). 

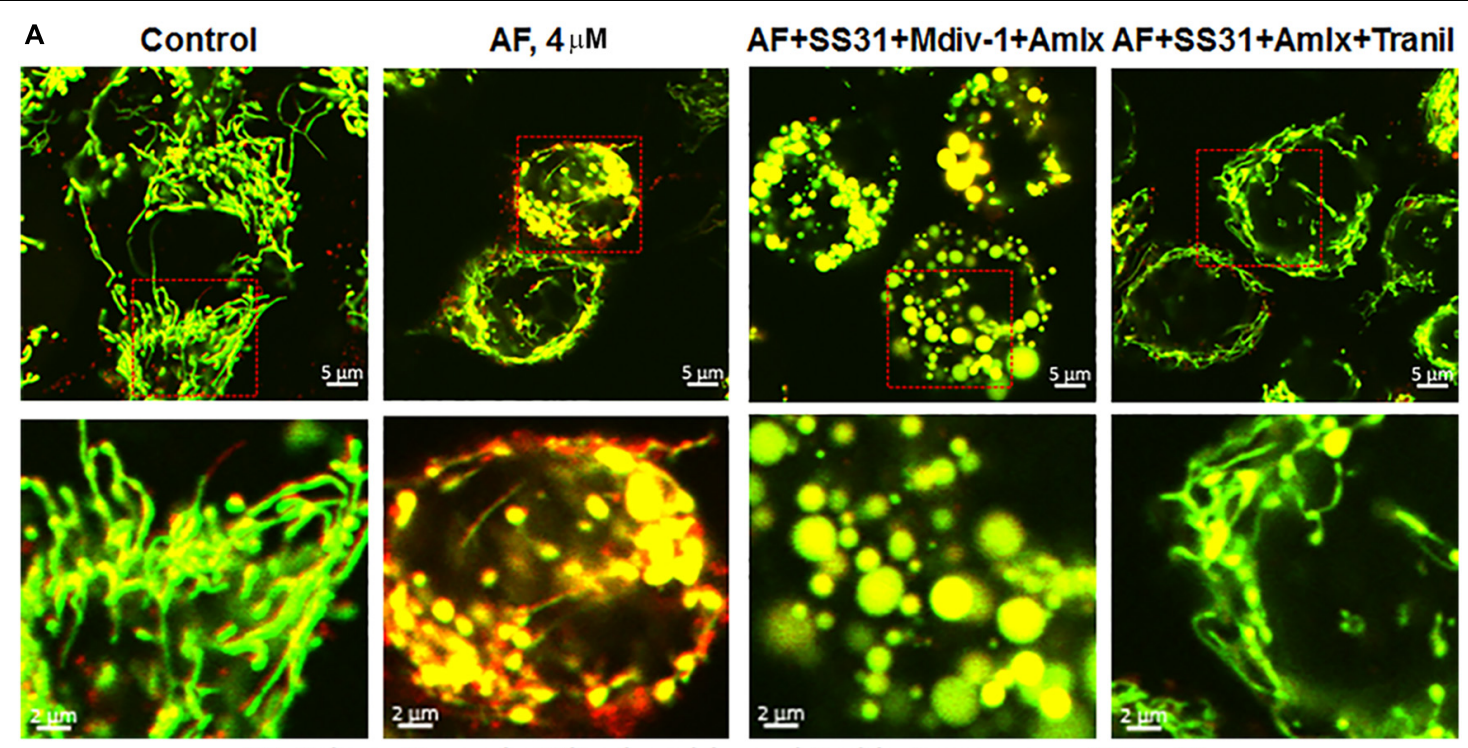

Mt-Keima: Green in Mitochondria and Red in Lysosomes; ARPE-19

B Control
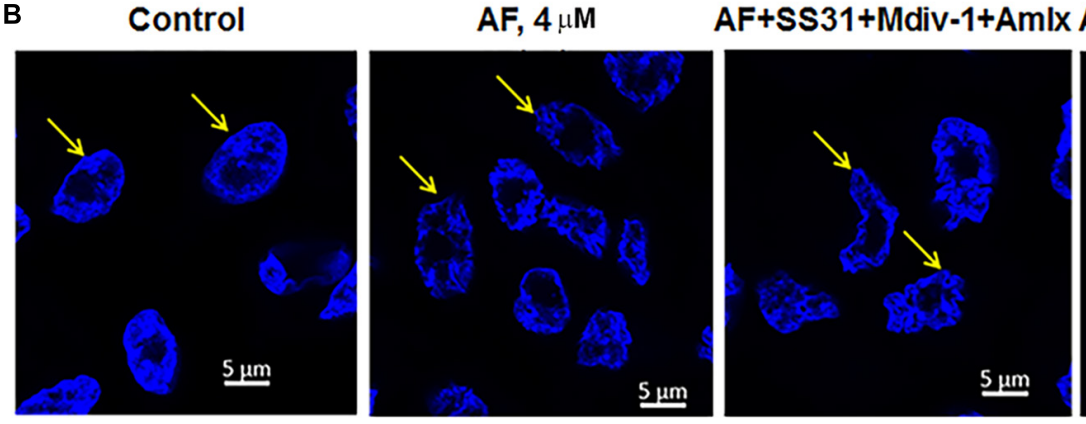

ARPE-19, Nuclei - Hoescht

C

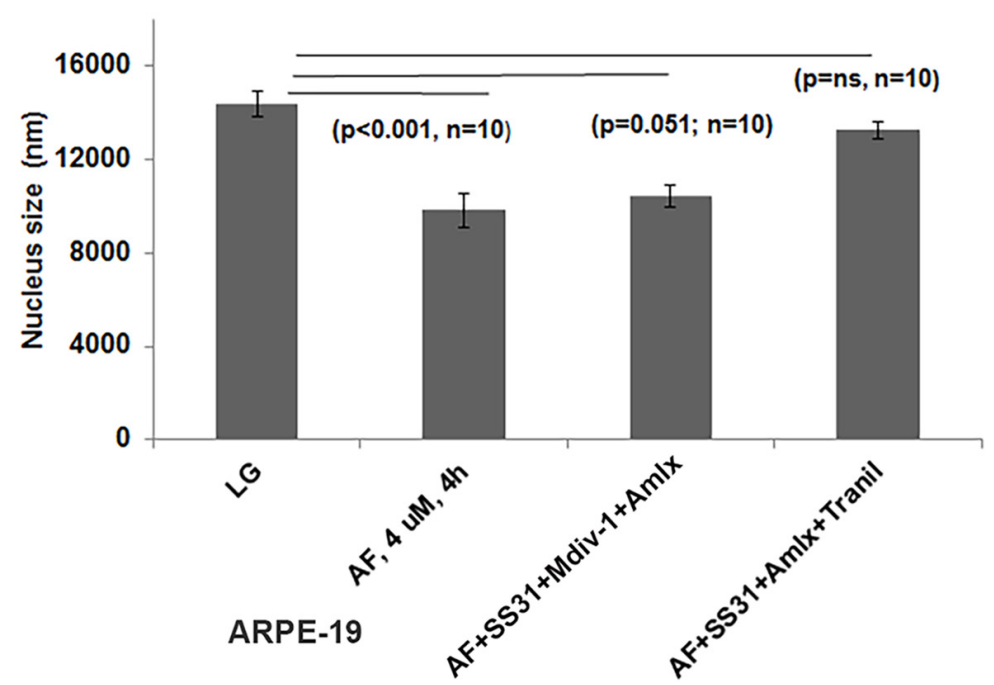

Concentrations:

SS31 - $4 \mu \mathrm{M}$;

Midv-1 - $56.6 \mu \mathrm{M}$;

Amlx - $4 \mu \mathrm{M}$;

Tranil - $56.5 \mu \mathrm{M}$

FIGURE 4 | Auranofin causes mitophagic flux and nuclear deformation in ARPE-19 cells. (A) AF treatment $(4 \mu \mathrm{M}, 4 \mathrm{~h})$ increases the level of red mt-Keima in ARPE-19 cells when compared to the control where green mt-Keima is predominantly observed. Combination treatment of ARPE-19 cells with SS31 + Midiv-1 + Amlexanox (added $2 \mathrm{~h}$ before AF) reduces red mt-Keima; however, the treatment also results in the formation of vesiculated (round and enlarged) mitochondria. Conversely, pretreatment with SS31 + Amlexanox + Tranilast prevents AF-induced mitophagy (reduced red mt-Keima); also seen as filamentous green network. A representative of $n=3$ is shown here at mag. $630 \times$. (B,C) AF causes nuclear deformation or chromatin shrinkage as shown by DAPI staining and reduced nuclear diameter compared to the control ARPE-19 (significant decrease, $p<0.001 \mathrm{AF}$ vs. Control; $n=10$ nuclei). Such nuclear changes are prevented by pre-treatment with a combination of SS31 + Amlexanox + Tranilast ( $p=n s$ vs. LG, control) but not with SS31 + Midiv-1 + Amlexanox $(p=0.051 ; n=10$ vs. LG, control). 


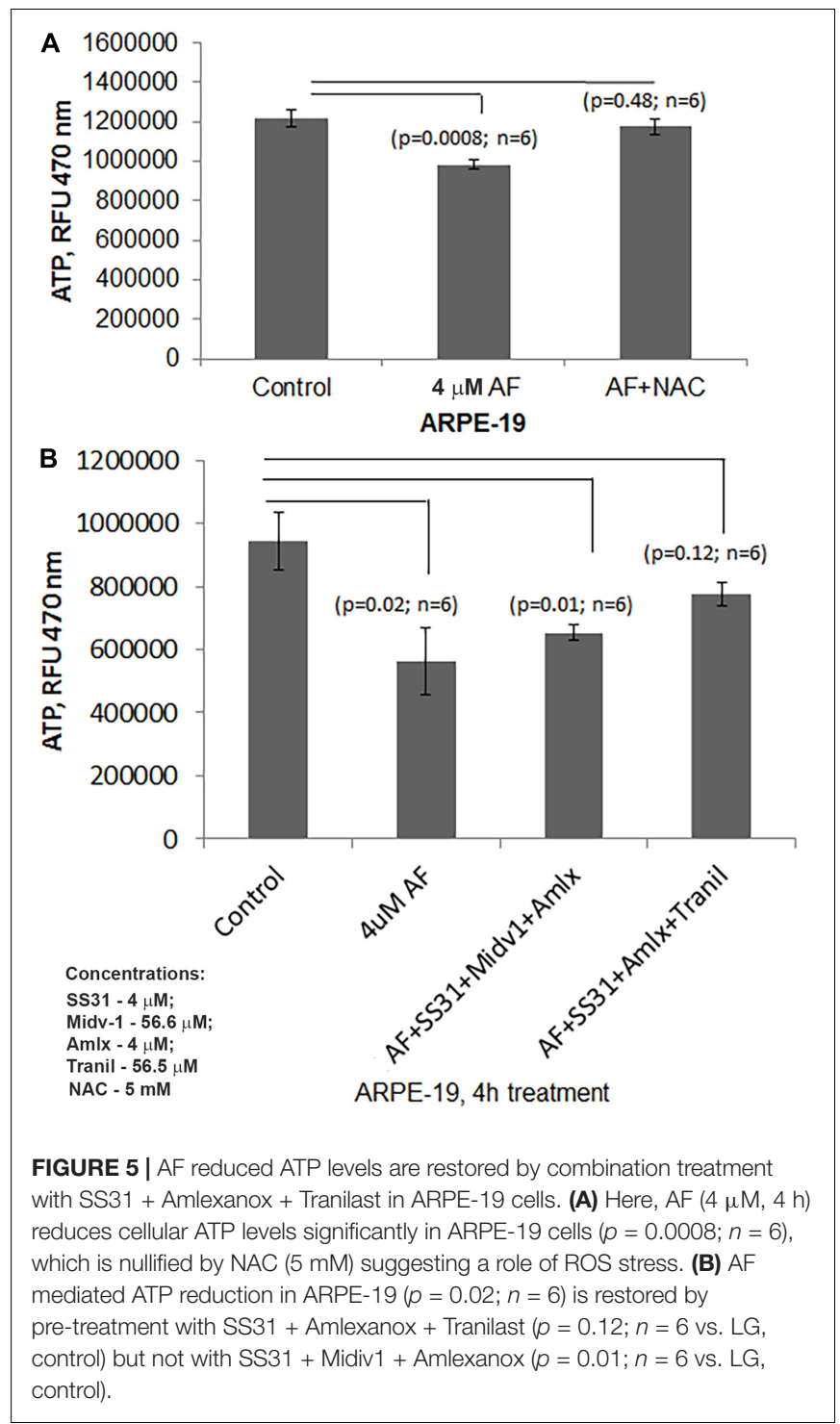

Therefore, the AF effect on ARPE-19 observed here may also be similar to defects in TXNIP and Trx-thiol redox signaling defects in DR to an extent (Di Carlo et al., 2012; Devi et al., 2013; Stephenson et al., 2018). RPE oxidative stress and mitochondrial dysfunction are also seen in AMD and RP, serious blinding retinal neurodegenerative diseases (Kaarniranta et al., 2019; Zhou et al., 2019). RPE is a fully differentiated cell; therefore, they cannot dilute or distribute damaged mitochondria to daughter cells. Therefore, an efficient removal of the damage mitochondria by mitophagy and biosynthesis of new mitochondria (mitogenesis) to maintain number and bioenergetics is critically important. In addition, lysosomal function needs to be preserve for its POS phagocytosis and visual pigment recycling. Mitophagy is a protective and survival mechanism for cell viability (Pareek and Pallanck, 2018); however, too much of a mitophagic flux could lead to lysosomal overloading, enlargement, and reduced capacity to digest proteolipids causing accumulation of undigested lipofuscin and drusens in Bruch's membrane in
AMD (Dias et al., 2018; Zhou et al., 2019). Similarly, the pathogenesis of genetic neurodegenerative diseases including RP also involves RPE mitochondrial dysfunction and photoreceptor demise. As mentioned before, photoreceptors are very active neurons, which maintain a large number of mitochondria for its bioenergetics (Shu and Dunaief, 2018; Kaarniranta et al., 2019). Therefore, not only in RPE cells, mitophagy defects, and bioenergetics failure in photoreceptors may also be involved in RP etiology. Nonetheless, the role of redox imbalance and mitophagy dysfunction in RP is yet to be fully understood.

Mitochondria are the powerhouse of the cell; therefore, their dysregulation leads to excess ROS production while ATP synthesis is blunted causing bioenergetics failure in neurodegeneration (Moreno et al., 2018). In addition, damaged mitochondria release their components into the cytosol, such as mtROS and oxidized mtDNA, which are recognized as DAMPs (damage associated molecular patterns) by the cytosolic PRRs including TLRs (Toll-like receptors) and NODlike NLRP3 inflammasomes (Zhao et al., 2019). Indeed, we demonstrate in this study that AF increases caspase-1 activity while that of the lysosomal enzyme cathepsin $L$ is reduced, indicating an autophagic/mitophagic flux to lysosome and its destabilization (Devi et al., 2013). Cathepsins and other lysosomal acid hydrolases are important for proper digestion of the ingested cargos and recycling (exocytosis of) the digested material as nutrients (Wilkins et al., 2017). Therefore, the removal of damaged mitochondria by mitophagy is critically important to reduce cellular ROS, inflammation and premature cell demise.

Two important cellular anti-oxidant systems are the Trxs and glutathione (Samie and $\mathrm{Xu}, 2014)$. The Trx/TrxR works with NADPH and peroxiredoxin 3 (Prx3) to scavenge hydrogen peroxide in mitochondria and to maintain a reduced state of cysteines in a protein (Ren et al., 2017). The GSH/GPX (particularly the mtGPX4 and cytosolic GPX4) are the sole enzymes to detoxify membrane lipid peroxidation and damage (Forred et al., 2017). Therefore, these two systems work together to maintain mitochondrial health and cellular function and, therefore, a defect in one of the two systems will lead to overburden and depletion of the anti-oxidant system in mitochondria, bioenergetics failure, and mitophagy-lysosome pathway dysregulation leading to demise (Samie and $\mathrm{Xu}, 2014$ ). Indeed, we show in this study that AF induces caspase-1 activation and cell death by pyroptosis in APRE-19 as NLRP3 and caspase- 1 inhibitors prevent AF-mediated cell death. In support, we also observed an increase in gasdermin D levels by AF. Caspase- 1 cleaves a $50-\mathrm{kD}$ gasdermin $\mathrm{D}$ to a $29-\mathrm{kD}$ pore forming $\mathrm{N}$-terminus fragment, which is inserted into the plasma membrane causing membrane leakage and cell death by pyroptosis (Dubois et al., 2019). Recently, a role for gasdermine E in pyroptosis was implicated (Jiang et al., 2019); therefore, we will perform further studies on the role of caspase- 1 and gasdermin isoforms in pyroptosis.

Furthermore, necrostatin 1 (necroptosis inhibitor) and ferrostatin 1 (ferroptosis inhibitor) are ineffective in blocking the AF-induced cell death in ARPE-19. Mitochondrial involvement 


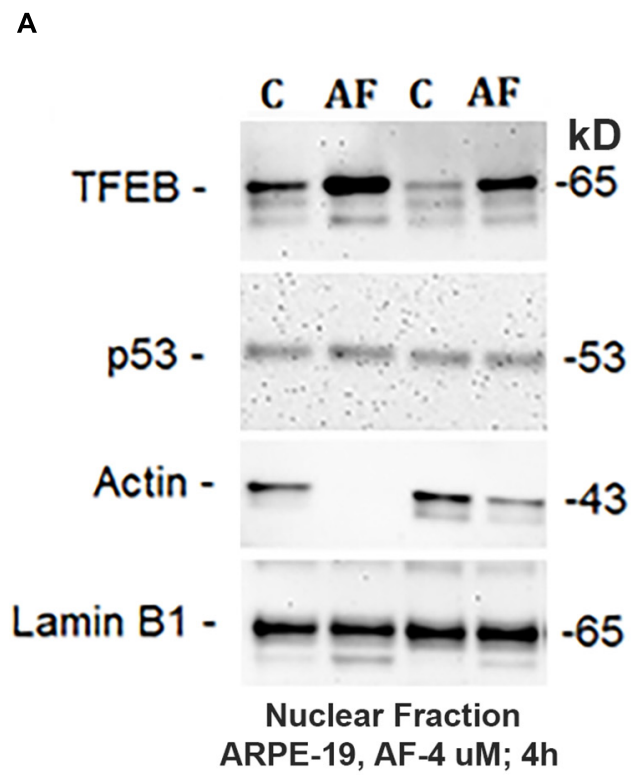

C

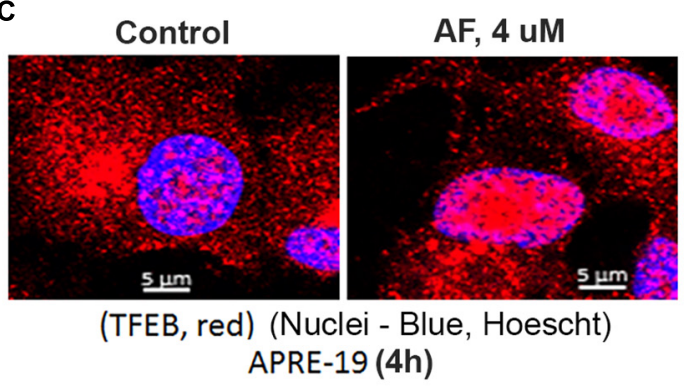

B
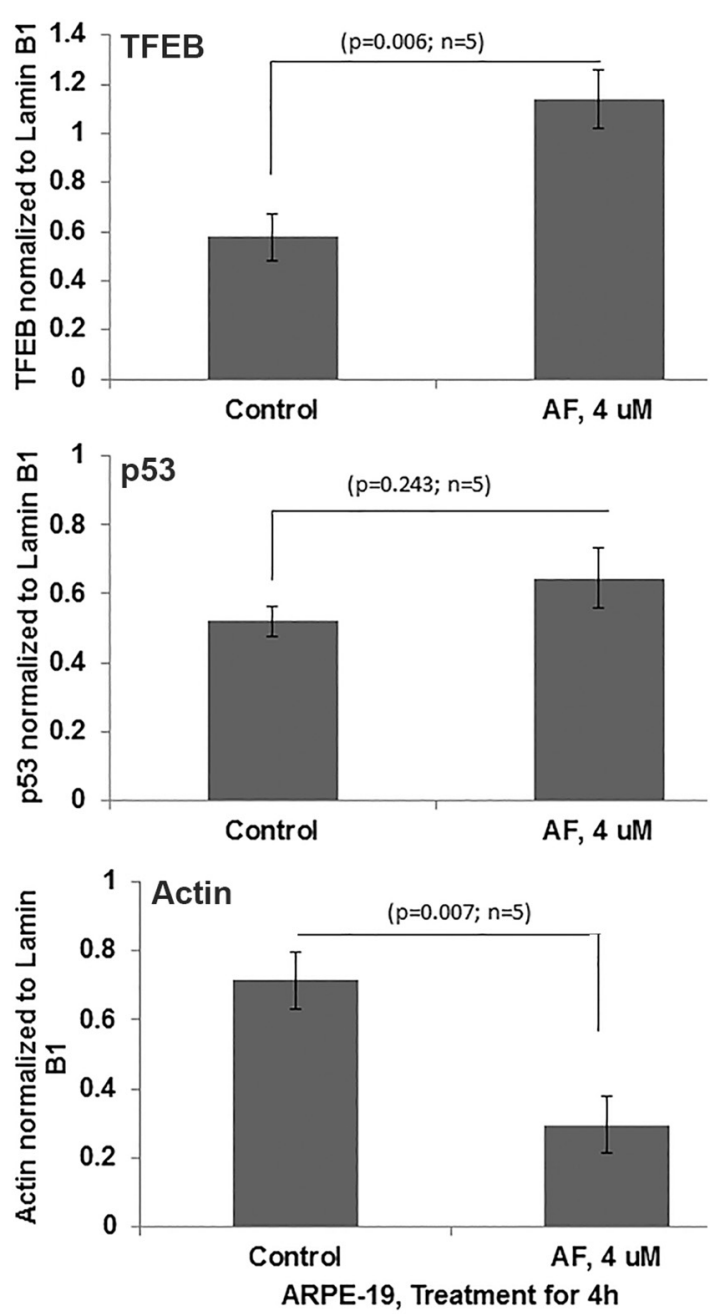

FIGURE 6 | AF increases nuclear TFEB level and reduces actin in ARPE-19. (A,B) AF treatment (4 $\mu$ M, 4 h) of ARPE-19 induces nuclear translocation of transcription factor TFEB significantly than in controls ( $p=0.006 ; n=5)$ on Western blots while that of the p53 is unchanged ( $p=0.245 ; n=5)$. Interestingly, the level of nuclear actin is significantly reduced $(p=0.007 ; n=5)$ than in controls. All proteins were normalized to nuclear lamin B1. (C) Similarly, nuclear translocation of TFEB after treatment with $\mathrm{AF}(4 \mu \mathrm{M}, 4 \mathrm{~h})$ is also seen when examined by confocal microscopy. A representative of $n=3$ is shown; mag. $630 \times$.

in this ARPE-19 death is also evident by inhibition of the mitochondrial dysfunction and cell death by a combination of SS31 (mitochondrial antioxidant), Amlexanox (inhibits TBK1 and normalize mitophagic flux) and tranilast (which inhibits NLRP3). Therefore, these three drug combinations may be useful in the treatment of mitochondria-associated neurodegenerative diseases particularly in DR as well as in AMD and PR.

One of the important factors in the mitochondrial quality control is coordinated regulation of mitophagy (removal of damaged parts of mitochondria by autophagy as mentioned earlier) and mitogenesis (synthesis of new mitochondrial and fusion with existing mitochondria) to maintain an optimal mitochondrial number and efficient bioenergetics (Maiorino et al., 2018). One transcription factor that is critical for lysosome and autophagy gene expression is TFEB (Huang et al., 2018). TFEB is also involved in the mitochondrial biogenesis via induction of PGC1 $\alpha$, another important transcription factor for nuclear-encoded mitochondrial gene expression (Ni et al., 2015; Pan et al., 2019). The fact that we observe TFEB translocation to the nucleus upon AF addition to ARPE-19 indicates lysosomal destabilization. Interestingly, the nuclear actin is downregulated suggesting nuclear chromatin organization may be disturbed and, therefore, transcriptional activity may be altered (Salma et al., 2015; Lammerding and Wolf, 2016). Further studies are needed to clarify this observation. TFEB is phosphorylated at multiple sites, including Ser122, Ser142, and Ser211, by mTORC1 at the lysosomal membrane and traps TEFB in the cytosol via an interaction with 14-3-3 scaffold proteins (Hatch and Hetzer, 2016). Conversely, TFEB nuclear translocation is regulated by mucolipin 1/TRPML1, a lysosomal calcium and divalent metal ion channel (Napolitano et al., 2018). Transient release of lysosomal calcium locally by TRPML1 activates calcineurin, a protein phosphatase, which dephosphorylates TFEB sites targeted by mTORC1 and releases from 14-3-3, 

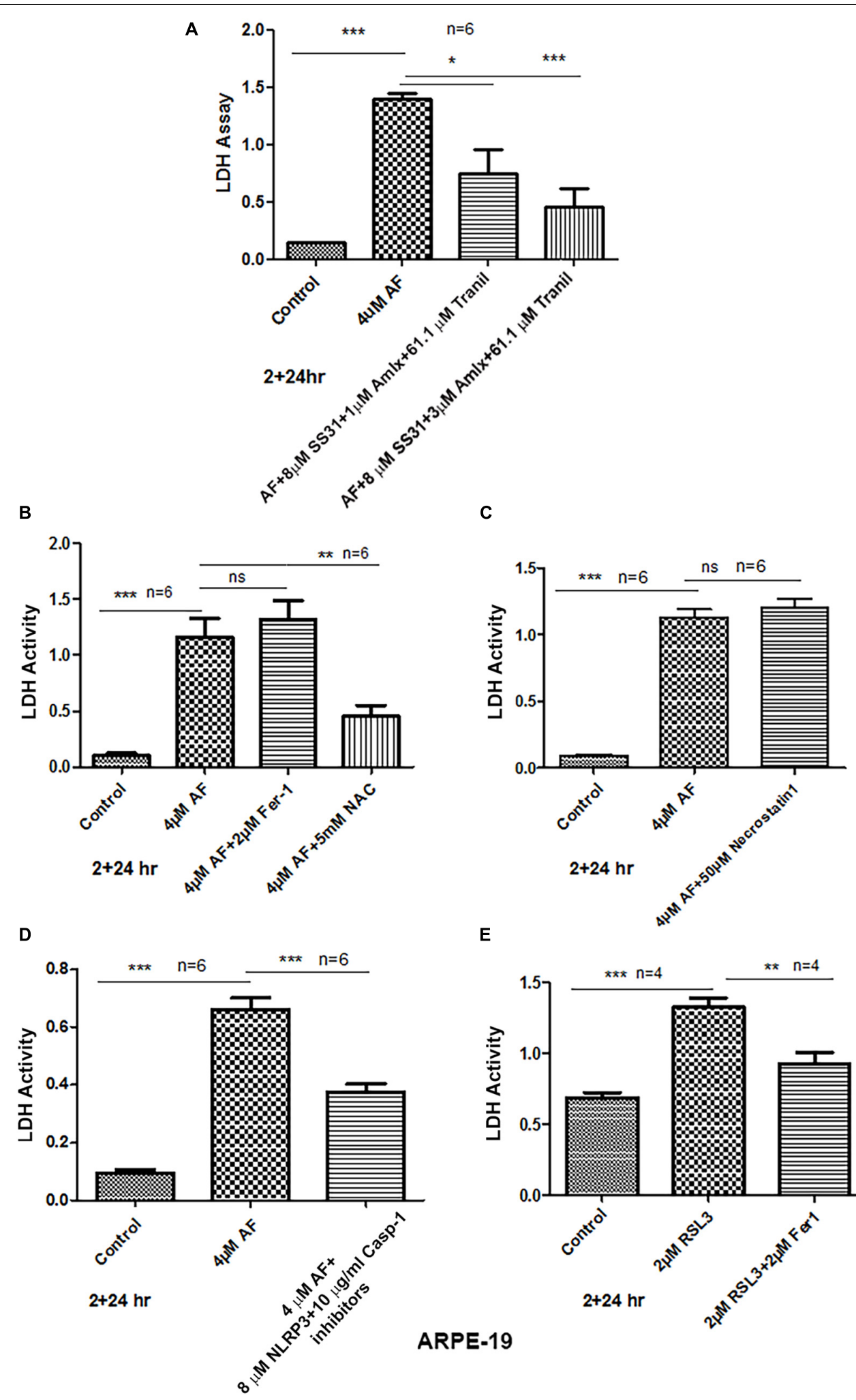

E

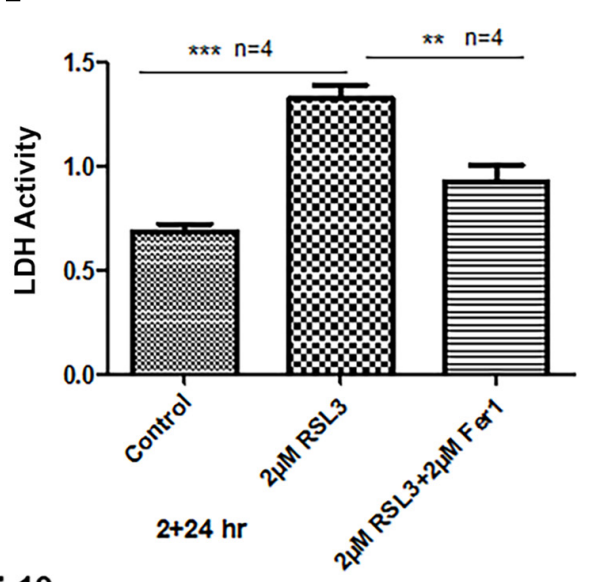

ARPE-19

FIGURE 7| AF induces ARPE-19 death by pyroptosis. (A) AF (4 $\mu \mathrm{M}, 24 \mathrm{~h})$ treatment of ARPE-19 cells induces cell death as measured by cytosolic LDH release in the culture media. AF effect is significantly countered by addition ( $2 \mathrm{~h}$ before AF) of three drugs combination SS31 + Amlexanox + Tranilast. (B) AF-induced LDH release is not reduced by ferrostatin-1 (inhibitor of ferroptosis, an iron-dependent cell death) but with NAC (N-acetylcysteine), suggesting the role of anti-oxidants. (C) Similarly, necrostatin-1 (an inhibitor of necroptosis) was without an effect on AF-mediated LDH release. (D) Conversely, AF-mediated LDH release is significantly (Continued) 
FIGURE 7 | Continued

reduced by a combination of NLRP3 (MCC950, $8 \mu \mathrm{M}$ ) and caspase-1 (Ac-YVAD-cmk, $10 \mu \mathrm{g} / \mathrm{ml}$ ) inhibitors (pyroptosis inhibitors). Symbols, * $p<0.05 ;{ }^{* *} p<0.001$; ${ }^{* * *} p<0.0001 ; n=6$ for all experiments. (E) Lastly, ferroptosis inducer RSL3 (an inhibitor GPX4) causes LDH release (ferroptotic cell death), which is significantly reduced by ferrostatin-1, further indicating that AF-mediated ARPE-19 cell death is due to inflammatory pyroptosis.

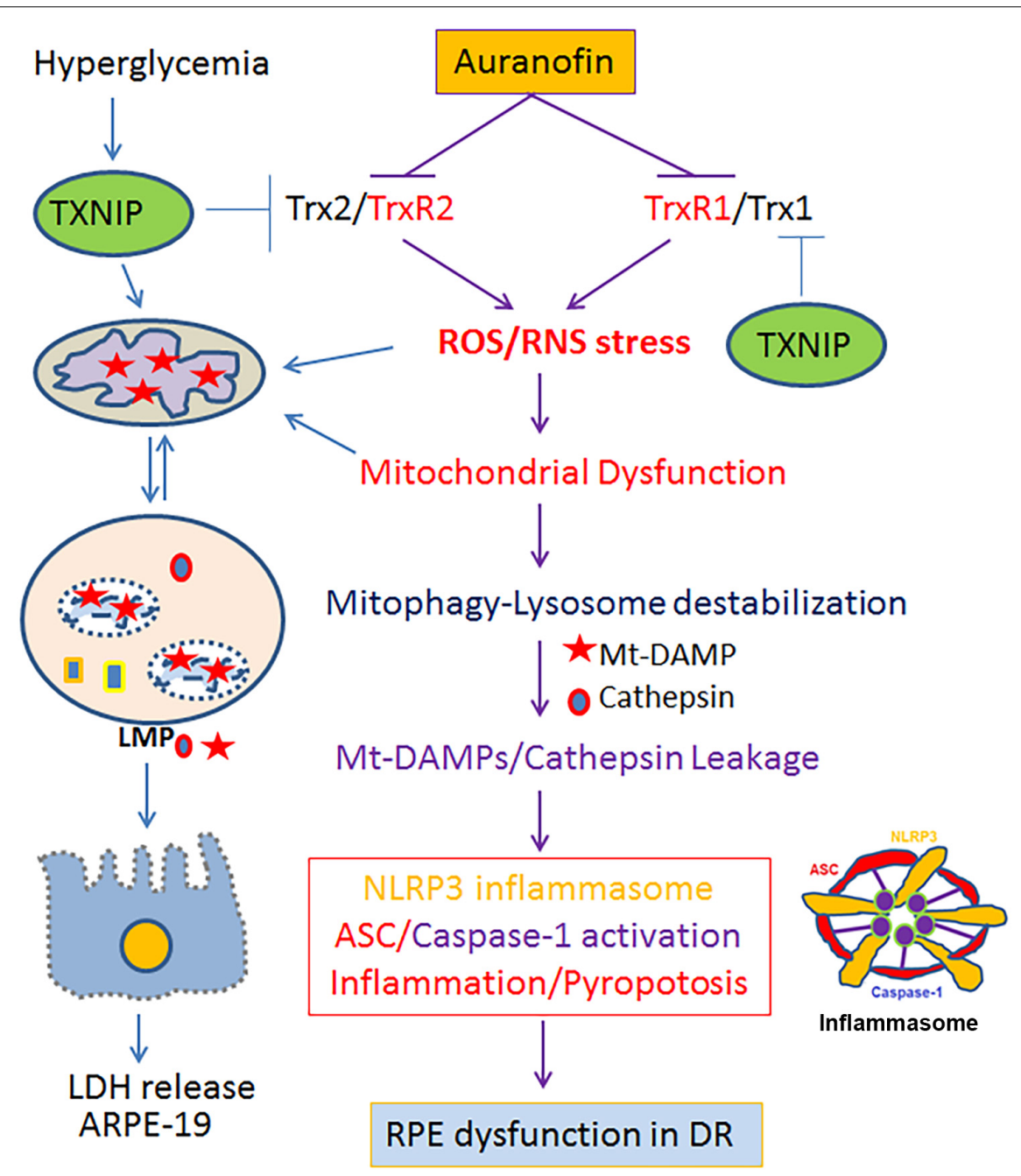

FIGURE 8 | A schematic diagram depicting a potential role for the Trx/TrxR redox pathway in oxidative stress and mitochondria-lysosome axis dysfunction in RPE. Inhibition of the Trx/TrxR pathway either by auranofin on TrxR or by TXNIP on Trx under hyperglycemia (Devi et al., 2019) may lead to cellular oxidative stress, mitochondrial dysfunction, altered mitophagic flux, lysosomal damage and pro-inflammatory cell death in DR as well as in other chronic retinal neurodegenerative diseases such as AMD and RP. A combination therapy strengthening the Trx/TrxR redox system and normalizing the mitochondrial-lysosomal axis may be a potential therapeutic approach to prevent progression of various neurodegenerative diseases.

thereby translocating to the nucleus (Napolitano et al., 2018). Nonetheless, TRPML1 is also regulated by PI3,5 bisphosphate in the lysosomal membrane, which is synthesized by PIKFYVE kinase (Di Paola and Medina, 2019). Therefore, these kinases and phosphatases may also involve in the regulation of lysosomal function, autophagy/mitophagy flux, exocytosis, and mitochondrial biogenesis. Indeed, we observe that apilimod (Medina et al., 2015), an inhibitor of PIKFYVE, causes lysosomal enlargement and cathepsin L downregulation, while ML-SA1 (Medina et al., 2015), an activator of TRPML1, causes lysosome biogenesis and increase cathepsin L level (unpublished data).
To what extent the Trx-TrxR and redox signaling regulates these kinases and phosphatases are yet to be determined in RPE and retinal neurodegenerative diseases. Nonetheless, most chronic neurodegenerative diseases involve dysregulation of mitochondrial function and lysosomal defects leading to bioenergetics failure and accumulation of undigested materials inside cells as well as extracellularly, particularly for RPE cells because they are involved in the degradation of melanosomes and phagocytosis of photoreceptor outer segments (POS) daily (Isobe et al., 2019). Thus, an accumulation of undigested oxidized lipoproteins with lysosomes will reduce lysosomal 
enzyme activities and enhance exocytosis of undigested material to the Bruch's membrane (a combined basement membrane of the RPE and endothelium of the choriocapillaris), which form Drusens, yellow deposits under the retina (Isobe et al., 2019). Such an event may cause RPE dysregulation and photoreceptor dysfunction in chronic retinal neurodegenerative diseases.

\section{CONCLUSION}

The present findings suggest that the Trx/TrxR redox system is critical for reducing oxidative stress, normalizing mitophagic flux, lysosome function, and prevention of pyroptotic cell death in the RPE, and therefore, in preventing retinal neurodegenerative diseases. Furthermore, the effectiveness of the therapy with three drug combinations targeting the mitochondrial ROS, mitophagic flux and NLRP3 inflammasome $(\mathrm{SS} 31+$ Amlexanox + Tranilast, respectively) needs to be tested in retinal neurodegenerative diseases of animal models particularly in DR. This three drugs combination may also be beneficial in the treatment and prevention of retinal neurodegenerative diseases such as AMD and RP as they also encounter mitochondrial and lysosomal dysregulation in the etiology of the diseases (Warburton et al., 2007; Bergen et al., 2019). The present study was presented as a poster at the annual meeting of the ARVO April 28, 2019 - May 02, 2019 at Vancouver, Canada.

\section{REFERENCES}

Bapputty, R., Talahalli, R., Zarini, S., Samuels, I., Murphy, R., and Gubitosi-Klug, R. (2019). Montelukast prevents early diabetic retinopathy in mice. Diabetes 68 , 2004-2015. doi: 10.2337/db19-0026

Bergen, A. A., Arya, S., Koster, C., Pilgrim, M. G., Wiatrek-Moumoulidis, D., van der Spek, P. J., et al. (2019). On the origin of proteins in human drusen: the meet, greet and stick hypothesis. Prog. Retin. Eye Res. 70, 55-84. doi: 10.1016/j. preteyeres.2018.12.003

Biesemeier, A., Yoeruek, E., Eibl, O., and Schraermeyer, U. (2015). Iron accumulation in Bruch's membrane and melanosomes of donor eyes with agerelated macular degeneration. Exp. Eye Res. 137, 39-49. doi: 10.1016/j.exer. 2015.05.019

Booty, L. M., Gawel, J. M., Cvetko, F., Caldwell, S. T., Hall, A. R., Mulvey, J. F., et al. (2019). Selective disruption of mitochondrial thiol redox state in cells and in vivo. Cell Chem. Biol. 26, 449-461. doi: 10.1016/j.chembiol.2018.12.002

Campbell, M. I, and Humphries, P. (2012). The blood-retina barrier: tight junctions and barrier modulation. Adv. Exp. Med. Biol. 763, 70-84. doi: 10.1007/978-14614-4711-5_3

Campbell, M. D., Duan, J., Samuelson, A. T., Gaffrey, M. J., Merrihew, G. E., Egertson, J. D., et al. (2019). Improving mitochondrial function with SS-31 reverses age-related redox stress and improves exercise tolerance in aged mice. Free Radic. Biol. Med. 134, 268-281. doi: 10.1016/j.freeradbiomed.2018.12.031

Caspard, H., Jabbour, S., Hammar, N., Fenici, P., Sheehan, J. J., and Kosiborod, M. (2018). Recent trends in the prevalence of type 2 diabetes and the association with abdominal obesity lead to growing health disparities in the USA: an analysis of the NHANES surveys from 1999 to 2014. Diabetes Obes. Metab. 20, 667-671. doi: 10.1111/dom.13143

Cheloni, R., Gandolfi, S. A., Signorelli, C., and Odone, A. (2019). Global prevalence of diabetic retinopathy: protocol for a systematic review and meta-analysis. BMJ Open 9:e022188. doi: 10.1136/bmjopen-2018-022188

Chiquita, S., Rodrigues-Neves, A. C., Baptista, F. I., Carecho, R., Moreira, P. I., Castelo-Branco, M., et al. (2019). The retina as a window or mirror of the

\section{DATA AVAILABILITY STATEMENT}

All materials and data developed in this study will be made available upon request.

\section{AUTHOR CONTRIBUTIONS}

TY performed the cell assays, developed the methodologies, and wrote the Materials and Methods section and reviewed the manuscript. TD performed the Western blotting and immunohistochemistry. LS developed the concept, provided the guidance, and wrote the manuscript.

\section{FUNDING}

This work was supported by the grants EY023992 (LS), Research to Prevent Blindness (RPB to OVAS), and Core Grant P30 EY004068 (OVAS).

\section{SUPPLEMENTARY MATERIAL}

The Supplementary Material for this article can be found online at: https://www.frontiersin.org/articles/10.3389/fnins. 2019.01065/full\#supplementary-material

brain changes detected in Alzheimer's disease: critical aspects to unravel. Mol. Neurobiol. 56, 5416-5435. doi: 10.1007/s12035-018-1461-6

Country, M. W. (2017). Retinal metabolism: a comparative look at energetics in the retina. Brain Res. 1672, 50-57. doi: 10.1016/j.brainres.2017.07.025

Cunha-Vaz, J., Bernardes, R., and Lobo, C. (2011). Blood-retinal barrier. Eur. J. Ophthalmol. 21(Suppl. 6), S3-S9. doi: 10.5301/EJO.2010.6049

Devi, T. S., Hosoya, K., Terasaki, T., and Singh, L. P. (2013). Critical role of TXNIP in oxidative stress, DNA damage and retinal pericyte apoptosis under high glucose: implications for diabetic retinopathy. Exp. Cell Res. 319, 1001-1012. doi: 10.1016/j.yexcr.2013.01.012

Devi, T. S., Lee, I., Hüttemann, M., Kumar, A., Nantwi, K. D., and Singh, L. P. (2012). TXNIP links innate host defense mechanisms to oxidative stress and inflammation in retinal müller glia under chronic hyperglycemia: implications for diabetic retinopathy. Exp Diabetes Res. 2012:438238.

Devi, T. S., Somayajulu, M., Kowluru, R. A., and Singh, L. P. (2017). TXNIP regulates mitophagy in retinal Müller cells under high-glucose conditions: implications for diabetic retinopathy. Cell Death Dis. 8:e2777. doi: 10.1038/ cddis. 2017.190

Devi, T. S., Yumnamcha, T., Yao, F., Somayajulu, M., Kowluru, R. A., and Singh, L. P. (2019). TXNIP mediates high glucose-induced mitophagic flux and lysosome enlargement in human retinal pigment epithelial cells. Biol Open 8:bio038521. doi: 10.1242/bio.038521

Di Carlo, M I, Giacomazza, D., Picone, P., Nuzzo, D., and San, Biagio PL (2012). Are oxidative stress and mitochondrial dysfunction the key players in the neurodegenerative diseases? Free Radic. Res. 46, 1327-1338. doi: 10.3109/ 10715762.2012.714466

Di Paola, S., and Medina, D. L. (2019). TRPML1-/TFEB-dependent regulation of lysosomal exocytosis. Methods Mol. Biol. 1925, 143-144. doi: 10.1007/978-14939-9018-4_12

Dias, M. F., Joo, K., Kemp, J. A., Fialho, S. L., da Silva Cunha, A. Jr., Woo, S. J., et al. (2018). Molecular genetics and emerging therapies for retinitis pigmentosa: basic research and clinical perspectives. Prog. Retin. Eye Res. 63, 107-131. doi: 10.1016/j.preteyeres.2017.10.004 
Dubois, H., Sorgeloos, F., Sarvestani, S. T., Martens, L., Saeys, Y., Mackenzie, J. M., et al. (2019). Nlrp3 inflammasome activation and gasdermin D-driven pyroptosis are immunopathogenic upon gastrointestinal norovirus infection. PLoS Pathog 15:e1007709. doi: 10.1371/journal.ppat.1007709

Fivenson, E. M., Lautrup, S., Sun, N., Scheibye-Knudsen, M., Stevnsner, T., Nilsen, H., et al. (2017). Mitophagy in neurodegeneration and aging. Neurochem. Int. 109, 202-209. doi: 10.1016/j.neuint.2017.02.007

Forcina, G. C., and Dixon, S. J. (2019). GPX4 at the crossroads of lipid homeostasis and ferroptosis. Proteomics 19:e1800311. doi: 10.1002/pmic.201800311

Forred, B. J., Daugaard, D. R., Titus, B. K., Wood, R. R., Floen, M. J., Booze, M. L., et al. (2017). Detoxification of mitochondrial oxidants and apoptotic signaling are facilitated by thioredoxin-2 and peroxiredoxin-3 during hyperoxic injury. PLoS One 12:e0168777. doi: 10.1371/journal.pone.0168777

Fu, Z., Löfqvist, C. A., Liegl, R., Wang, Z., Sun, Y., Gong, Y., et al. (2018). Photoreceptor glucose metabolism determines normal retinal vascular growth. EMBO Mol. Med. 10, 76-90. doi: 10.15252/emmm.201707966

Graham, P. S., Kaidonis, G., Abhary, S., Gillies, M. C., Daniell, M., Essex, R. W., et al. (2018). Genome-wide association studies for diabetic macular edema and proliferative diabetic retinopathy. BMC Med. Genet. 19:71. doi: 10.1186/s12881018-0587-8

Handa, J. T., Bowes Rickman, C., Dick, A. D., Gorin, M. B., Miller, J. W., Toth, C. A., et al. (2019). A systems biology approach towards understanding and treating non-neovascular age-related macular degeneration. Nat. Commun. 10:3347. doi: 10.1038/s41467-019-11262-1

Hanus, J., Anderson, C., Sarraf, D., Ma, J., and Wang, S. (2016). Retinal pigment epithelial cell necroptosis in response to sodium iodate. Cell Death Discov. 2:16054. doi: 10.1038/cddiscovery.2016.54

Harper, M. T. (2019). Auranofin, a thioredoxin reductase inhibitor, causes platelet death through calcium overload. Platelets 30, 98-104. doi: 10.1080/09537104. 2017.1378809

Hatch, E. M., and Hetzer, M. W. (2016). Nuclear envelope rupture is induced by actin-based nucleus confinement. J. Cell Biol. 215, 27-36. doi: 10.1083/jcb. 201603053

Huang, Y., Jiang, H., Chen, Y., Wang, X., Yang, Y., Tao, J., et al. (2018). Tranilast directly targets NLRP3 to treat inflammasome-driven diseases. EMBO Mol. Med. 10:e8689. doi: 10.15252/emmm.201708689

Isobe, Y., Nigorikawa, K., Tsurumi, G., Takemasu, S., Takasuga, S., Kofuji, S., et al. (2019). PIKfyve accelerates phagosome acidification through activation of TRPML1 while arrests aberrant vacuolation independent of the $\mathrm{Ca}^{2+}$ channel. J. Biochem. 165, 75-84. doi: 10.1093/jb/mvy084

Ivanova, E., Alam, N. M., Prusky, G. T., and Sagdullaev, B. T. (2019). Bloodretina barrier failure and vision loss in neuron-specific degeneration. JCI Insight 5:126747. doi: 10.1172/jci.insight. 126747

Jiang, S., Gu, H., Zhao, Y., and Sun, L. (2019). Teleost gasdermin E Is cleaved by caspase 1, 3, and 7 and induces pyroptosis. J. Immunol. 203, 1369-1382. doi: 10.4049/jimmunol.1900383

Jun, S., Datta, S., Wang, L., Pegany, R., Cano, M., and Handa, J. T. (2019). The impact of lipids, lipid oxidation, and inflammation on AMD, and the potential role of miRNAs on lipid metabolism in the RPE. Exp. Eye Res. 181, 346-355. doi: 10.1016/j.exer.2018.09.023

Kaarniranta, K., Pawlowska, E., Szczepanska, J., Jablkowska, A., and Blasiak, J. (2019). Role of mitochondrial DNA damage in ROS-mediated pathogenesis of age-related macular degeneration (AMD). Int. J. Mol. Sci. 20:E2374. doi: 10.3390/ijms20102374

Lammerding, J., and Wolf, K. (2016). Nuclear envelope rupture: actin fibers are putting the squeeze on the nucleus. J. Cell Biol. 215, 5-8. doi: 10.1083/jcb. 201609102

Lill, R., Hoffmann, B., Molik, S., Pierik, A. J., Rietzschel, N., Stehling, O., et al. (2012). The role of mitochondria in cellular iron-sulfur protein biogenesis and iron metabolism. Biochim. Biophys. Acta 1823, 1491-1508. doi: 10.1016/ j.bbamcr.2012.05.009

Lin, M. T., and Beal, M. F. (2006). Mitochondrial dysfunction and oxidative stress in neurodegenerative diseases. Nature 443, 787-795. doi: 10.1038/nature 05292

Liu, H., Tang, J., Du, Y., Saadane, A., Samuels, I., Veenstra, A., et al. (2019). Transducin1, phototransduction and the development of early diabetic retinopathy. Invest. Ophthalmol. Vis. Sci. 60, 1538-1546. doi: 10.1167/iovs.1826433
Maiorino, M., Conrad, M., and Ursini, F. (2018). GPx4, lipid peroxidation, and cell death: discoveries, rediscoveries, and open issues. Antioxid. Redox. Signal. 29, 61-74. doi: 10.1089/ars.2017.7115

Manczak, M., Kandimalla, R., Yin, X., and Reddy, P. H. (2019). Mitochondrial division inhibitor 1 reduces dynamin-related protein 1 and mitochondrial fission activity. Hum. Mol. Genet. 28, 177-199. doi: 10.1093/hmg/ddy335

Medina, D. L., Di Paola, S., Peluso, I., Armani, A., De Stefani, D., Venditti, R., et al. (2015). Lysosomal calcium signalling regulates autophagy through calcineurin and TFEB. Nat. Cell Biol. 17, 288-299. doi: 10.1038/ncb3114

Moreno, M. L., Mérida, S., Bosch-Morell, F., Miranda, M., and Villar, V. M. (2018). Autophagy dysfunction and oxidative stress, two related mechanisms implicated in retinitis pigmentosa. Front. Physiol. 9:1008. doi: 10.3389/fphys. 2018.01008

Napolitano, G., Esposito, A., Choi, H., Matarese, M., Benedetti, V., Di Malta, C., et al. (2018). mTOR-dependent phosphorylation controls TFEB nuclear export. Nat. Commun. 9:3312. doi: 10.1038/s41467-018-05862-6

Ni, H. M., Williams, J. A., and Ding, W. X. (2015). Mitochondrial dynamics and mitochondrial quality control. Redox. Biol. 2015, 6-13. doi: 10.1016/j.redox. 2014.11.006

Oral, E. A., Reilly, S. M., Gomez, A. V., Meral, R., Butz, L., Ajluni, N., et al. (2017). Inhibition of IKK $\varepsilon$ and TBK1 improves glucose control in a subset of patients with type 2 diabetes. Cell Metab. 26, 157.e7-170.e7. doi: 10.1016/j.cmet.2017. 06.006

Pan, H. Y., Alamri, A. H., and Valapala, M. (2019). Nutrient deprivation and lysosomal stress induce activation of TFEB in retinal pigment epithelial cells. Cell Mol. Biol. Lett. 24:33. doi: 10.1186/s11658-019-0159-8

Pareek, G., and Pallanck, L. J. (2018). Inactivation of lon protease reveals a link between mitochondrial unfolded protein stress and mitochondrial translation inhibition. Cell Death Dis. 9:1168. doi: 10.1038/s41419-0181213-6

Perrone, L., Devi, T. S., Hosoya, K. I., Terasaki, T., and Singh, L. P. (2010). Inhibition of TXNIP expression in vivo blocks early pathologies of diabetic retinopathy. Cell Death Dis. 1:E65. doi: 10.1038/cddis.2010.42

Pronin, A., Pham, D., An, W., Dvoriantchikova, G., Reshetnikova, G., Qiao, J., et al. (2019). Inflammasome activation induces pyroptosis in the retina exposed to ocular hypertension injury. Front. Mol. Neurosci. 12:3. doi: 10.3389/fnmol.2019. 00036

Ren, X., Zou, L., Zhang, X., Branco, V., Wang, J., Carvalho, C., et al. (2017). Redox signaling mediated by thioredoxin and glutathione systems in the central nervous system. Antioxid. Redox. Signal. 27, 989-1010. doi: 10.1089/ars.2016. 6925

Salma, N., Song, J. S., Arany, Z., and Fisher, D. E. (2015). Transcription factor Tfe3 directly regulates Pgc-1alpha in muscle. J. Cell. Physiol. 230, 2330-2336. doi: $10.1002 /$ jcp. 24978

Samie, M. A., and Xu, H. (2014). Lysosomal exocytosis and lipid storage disorders. J. Lipid Res. 55, 995-1009. doi: 10.1194/jlr.R046896

Settembre, C., and Medina, D. L. (2015). TFEB and the CLEAR network. Methods Cell Biol. 126, 45-62. doi: 10.1016/bs.mcb.2014.11.011

Shu, W., and Dunaief, J. L. (2018). Potential treatment of retinal diseases with iron chelators. Pharmaceuticals 11:E112. doi: 10.3390/ph11040112

Singh, L. P., and Perrone, L. (2013). Thioredoxin interacting protein (TXNIP) and pathogenesis of diabetic retinopathy. J. Clin. Exp. Ophthalmol. 4:287.

Spencer, W. J., Ding, J. D., Lewis, T. R., Yu, C., Phan, S., Pearring, J. N., et al. (2019). PRCD is essential for high-fidelity photoreceptor disc formation. Proc. Natl. Acad. Sci. U.S.A. 116, 13087-13096. doi: 10.1073/pnas.1906421116

Stephenson, J., Nutma, E., van der Valk, P., and Amor, S. (2018). Inflammation in CNS neurodegenerative diseases. Immunology 154, 204-219. doi: 10.1111/imm. 12922

Tarchick, M. J., Cutler, A. H., Trobenter, T. D., Kozlowski, M. R., Makowski, E. R., Holoman, N., et al. (2019). Endogenous insulin signaling in the RPE contributes to the maintenance of rod photoreceptor function in diabetes. Exp. Eye Res. 180, 63-74. doi: 10.1016/j.exer.2018.11.020

Warburton, S., Davis, W. E., Southwick, K., Xin, H., Woolley, A. T., Burton, G. F., et al. (2007). Proteomic and phototoxic characterization of melanolipofuscin: correlation to disease and model for its origin. Mol. Vis. 13, 318-329.

Wilkins, H. M., Weidling, I. W., Ji, Y., and Swerdlow, R. H. (2017). Mitochondriaderived damage-associated molecular patterns in neurodegeneration. Front. Immunol. 8:508. doi: 10.3389/fimmu.2017.00508 
Xia, T., and Rizzolo, L. J. (2017). Effects of diabetic retinopathy on the barrier functions of the retinal pigment epithelium. Vis. Res. 139, 72-81. doi: 10.1016/ j.visres.2017.02.006

Yoshida, M., Minagawa, S., Araya, J., Sakamoto, T., Hara, H., Tsubouchi, K., et al. (2019). Involvement of cigarette smoke-induced epithelial cell ferroptosis in COPD pathogenesis. Nat. Commun. 10:3145. doi: 10.1038/s41467-019-10 991-7

Yoshihara, E., Masaki, S., Matsuo, Y., Chen, Z., Tian, H., and Yodoi, J. (2014). Thioredoxin/Txnip: redoxisome, as a redox switch for the pathogenesis of diseases. Front. Immunol. 4:514. doi: 10.3389/fimmu.2013.00514

Zhao, Y., Sun, X., Hu, D., Prosdocimo, D. A., Hoppel, C., Jain, M. K., et al. (2019). ATAD3A oligomerization causes neurodegeneration by coupling mitochondrial fragmentation and bioenergetics defects. Nat. Commun. 10:1371. doi: 10.1038/s41467-019-09291-x
Zhou, B. I, Liu, J. II, Kang, R. III, Klionsky, D. J. IV, Kroemer, G. V, and Tang, D. VI (2019). Ferroptosis is a type of autophagy-dependent cell death. Semin. Cancer Biol. . doi: 10.1016/j.semcancer.2019.03.002 [Epub ahead of print].

Conflict of Interest: The authors declare that the research was conducted in the absence of any commercial or financial relationships that could be construed as a potential conflict of interest.

Copyright (c) 2019 Yumnamcha, Devi and Singh. This is an open-access article distributed under the terms of the Creative Commons Attribution License (CC BY). The use, distribution or reproduction in other forums is permitted, provided the original author(s) and the copyright owner(s) are credited and that the original publication in this journal is cited, in accordance with accepted academic practice. No use, distribution or reproduction is permitted which does not comply with these terms. 